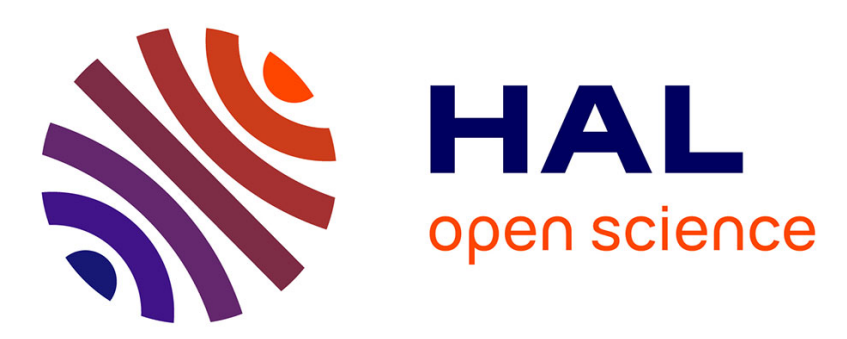

\title{
Accuracy study and validation techniques for fast beam-based monostatic scattering computations
}

Christine Letrou, Mossaab Hariz, Amir Boag

\section{To cite this version:}

Christine Letrou, Mossaab Hariz, Amir Boag. Accuracy study and validation techniques for fast beam-based monostatic scattering computations. ICEAA 2019: 21st International Conference on Electromagnetics in Advanced Applications, Sep 2019, Granada, Spain. pp.0824-0827, 10.1109/ICEAA.2019.8878975 . hal-02452844

\section{HAL Id: hal-02452844 \\ https://hal.science/hal-02452844}

Submitted on 23 Jan 2020

HAL is a multi-disciplinary open access archive for the deposit and dissemination of scientific research documents, whether they are published or not. The documents may come from teaching and research institutions in France or abroad, or from public or private research centers.
L'archive ouverte pluridisciplinaire HAL, est destinée au dépôt et à la diffusion de documents scientifiques de niveau recherche, publiés ou non, émanant des établissements d'enseignement et de recherche français ou étrangers, des laboratoires publics ou privés. 


\section{Accuracy Study and Validation Techniques for Fast Beam-Based Monostatic Scattering Computations}

\author{
Christine Letrou \\ SAMOVAR Lab. \\ Télécom SudParis, IP Paris \\ Evry, France \\ christine.letrou@telecom-sudparis.eu
}

\author{
Mossaab Hariz \\ Télécom SudParis, IP Paris \\ Evry, France \\ mossaab.hariz@telecom-sudparis.eu
}

\author{
Amir Boag \\ School of Electrical Engineering \\ Tel Aviv University \\ Tel Aviv, Israel \\ boag@ tauex.tau.ac.il
}

\begin{abstract}
A Gaussian beam shooting algorithm based on Gabor frame decomposition is proposed for fast monostatic scattering computations in large multipath environments. Formulation and specific features of the method are outlined. Complexity and accuracy of the algorithm are discussed and illustrated by numerical results. A validation strategy based on 2D-to-3D conversion is explored, due to the large electrical size of the problems to be solved with this method.
\end{abstract}

Index Terms-Gaussian beam, Gabor frame, monostatic scattering, Physical Optics, Method of Moments

\section{INTRODUCTION}

Scattering by objects or complex environments which are large with respect to the wavelength, and involve multiple internal reflections, is a challenge for simulation methods. Frame based Gaussian Beam Shooting (GBS) has been recently proposed as an alternative to Physical Optics (PO) based techniques (including Iterative PO and Multilevel PO) and ray-based methods (e.g. Shooting and Bouncing Rays) for monostatic scattering computations [1]. The proposed algorithm takes advantage of the Gaussian beam (GB) limited spectral and transverse spatial extents, which lead to dramatic limitation of the number of beams involved in monostatic RCS computations.

In the first part of this paper, the algorithm is presented in the context of scattering by urban type multipath environments. Its complexity is discussed in the second part of the paper, and analyzed with respect to frame parameters, especially the beamwidth to wavelength ratio. In the third part of the paper, the accuracy of the method is analyzed as a function of mainly two types of ratios involving the beamwidth, the one to wavelength, and the other to geometrical characteristic dimensions of the scattering environment. For this analysis, Physical Optics (PO) can be used as reference method only for elementary or not too large cases. For more complex configurations involving more internal reflexions, the validity of two-dimensional (2D) to three-dimensional (3D) conventional conversion formulas will be discussed, and the paper will propose some improvements to those. With such conversion, 2D scattering computations by the Method of Moments can then be used to validate GBS computations for 3D scattering problems.

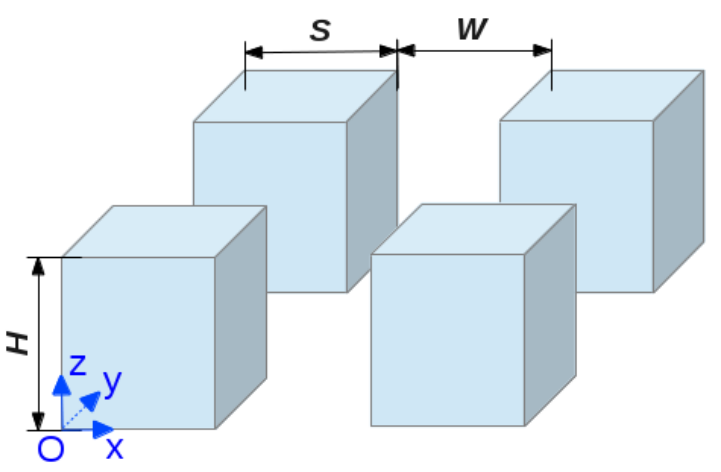

Fig. 1. Scattering environment scenario.

\section{FORMULATION}

\section{A. Frame based SCS computation}

The monostatic Scattering Cross Section (SCS), expressed in $\mathrm{dBsm}$, in the $\gamma$ direction, is defined as :

$$
S C S(\gamma)=20 \log 10\left(r_{o b s}\left|\varphi_{s}(\gamma) / \varphi_{i}(-\gamma)\right|\right)
$$

where $\varphi_{s}(\gamma)$ is the field of the scattered plane wave (PW) propagating in the direction $\gamma$ when a PW propagating in the opposite direction, with its field denoted $\varphi_{i}(-\gamma)$, is incident on the scattering object or environment.

The GBS algorithm relies on the equivalence theorem and on a PO-type approximation to define source fields: the incident field distribution on a surface outside the scattering object is taken as the source field. For a scattering environment of the type presented in Fig. 1, composed of reflecting blocks with planar walls, the smallest rectangular cuboid enclosing the object is used to support these source fields. The planes containing the six faces of this cuboid are indexed by $e \in E$, and denoted $P_{e}$.

Scattering computation with a frame based GBS method relies on the representation of incident fields as superpositions of Gaussian window distributions in the $P_{e}$ planes, via frame decomposition. Each of the Gaussian window distributions radiates in the form of a GB which undergoes one or multiple reflexions by block faces, either exterior ones, in one of the $P_{e}$, or inside the scattering environment. Reflexion transforms 
a GB into another GB, within the paraxial approximation. The field scattered outward is finally obtained as a superposition of the far fields of the transformed GBs. For the sake of simplicity, we shall consider an acoustic problem (scalar fields) with hard boundary condition.

\section{B. GBS formulation}

A frame Gaussian window is of the form

$$
w_{m n}(x)=w(x-m \bar{x}) e^{i n \bar{k}_{x}(x-m \bar{x})}
$$

with $w(x)=\sqrt{\sqrt{2} / L} e^{-\pi \frac{x^{2}}{L^{2}}}$. The integer frame index $m$ and the frame parameter $\bar{x}$ (resp. $n$ and $\bar{k}_{x}$ ) are the spatial (resp. spectral) translation index and translation step. For the set $\left\{w_{m n},(m, n) \in \mathbb{Z}^{2}\right\}$ to be a frame, it must be redundant, i.e. $\bar{k}_{x} \bar{x}=2 \pi \nu$ with $\nu<1$. In a plane spanned by the $x$ and $y$ variables, a frame window is obtained as the product of frame windows along $x$ and $y: w_{\mu}(x, y)=w_{m n}(x) w_{p q}(y)$ with $\boldsymbol{\mu}=(m, n, p, q) \in \mathbb{Z}^{4}$.

The decomposition coefficients of a planar field distribution on a frame is obtained by projection of the distribution on "dual frame" windows, which are proportional to frame windows for redundant enough frames $(\nu<0.33)$. The field distribution in the plane $P_{e}(O, x, y)$ of a PW with wave vector $\boldsymbol{k}_{i}$, denoted $\varphi_{\circ}(x, y)$, is easily decomposed on a frame in this plane:

$$
\begin{gathered}
\varphi_{\circ}(x, y)=\sum_{\mu \in \mathbb{Z}^{4}} a_{\mu} w_{\mu}(x, y) \\
\text { with } a_{\mu}=\nu e^{-i\left(m n \bar{x} \bar{k}_{x}+p q \bar{y} \bar{k}_{y}\right)} \widetilde{w}_{-m, n,-p, q}\left(k_{i, x}, k_{i, y}\right)
\end{gathered}
$$

where $\widetilde{w}$ is the (Gaussian) spectrum of the $w$ Gaussian window.

\section{Image beams}

Fig. 2 presents the situation where a GB radiated by a $w_{\mu}$ frame window in a source plane is reflected by the $\left(O_{P}, \hat{x}_{P}, \hat{y}_{P}, \hat{z}_{P}\right)$ plane. The reflected field can then be considered as being radiated by an "image frame" Gaussian window $\widetilde{w}_{\mu_{I}}$, in the $\left(O_{I}, \hat{x}_{I}, \hat{y}_{I}, \hat{z}_{I}\right)$ plane shown on the figure, the image frame being defined by the same parameters as the source plane frame. The frame index $\boldsymbol{\mu}_{I}$ is related to the incident frame window index $\boldsymbol{\mu}$ as follows: $\boldsymbol{\mu}_{I}=\left(m_{I}, n_{I}, p_{I}, q_{I}\right)=(-m,-n, p, q)$.

Once a GB has been tracked through successive reflexions until scattering out of the environment, its field can be easily computed, knowing the coordinate system of the last created image plane and the total number of reflexions. In cases when the reflexion coefficients depend on angles of incidence, information about the successive planes of reflexion is also required to compute the product of reflexion coefficients for all successive reflexions.

\section{Beam selection}

The spatial and spectral extents of a $w_{m n}$ frame window are defined with respect to a given threshold level. Taking the same threshold in both domains, the half-widths at level $\epsilon$ are given

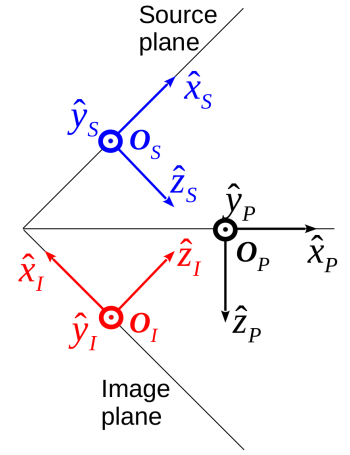

Fig. 2. GB reflexion: coordinate systems for image frame definition.

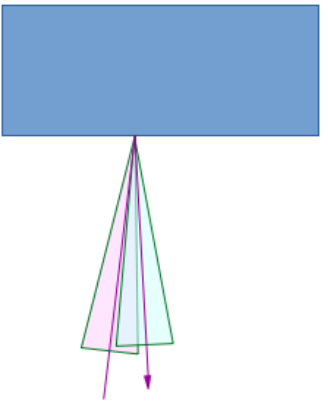

(a) Eligible beam

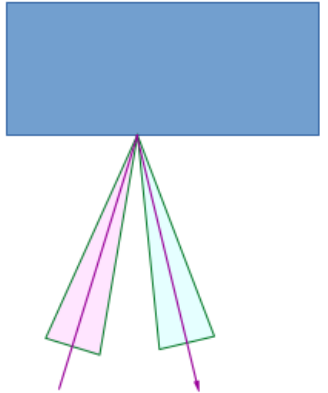

(b) Non-eligible beam
Fig. 3. Cones of horizontal directions of a scattered GB PWS and of directions opposite to the initial incident GB PWS.

by: $L^{\epsilon}=L \sqrt{-\ln (\epsilon) / \pi}$ (spatial) and $\Omega^{\epsilon}=\frac{2}{L} \sqrt{-\pi \ln (\epsilon)}$ (spectral). This threshold level can also be used to determine the transverse extent of a propagating GB.

Due to their limited spectral extent, not all GBs propagating out of the environment contribute to monostatic SCS. Those with no common direction of propagation between their plane wave spectrum (PWS) and the directions opposite to those in the plane wave spectrum of their initial source window $w_{\mu_{e}}$ in $P_{e}$ cannot contribute. Cases of contributing and non contributing beams are presented in Fig. 3 in the simple case when an initial GB with "horizontal" axis is directly reflected outward by "vertical" $P_{e}$.

\section{ALGORITHM AND COMPLEXITY}

\section{A. Algorithm outline}

In order to take advantage of GB properties, the first part of the algorithm is devoted to characterizing the scattering process via frame based GB tracking.

The GB tracking process is performed successively for all the $P_{e}$ planes, and consists for each of them in determining which frame windows are at the origin of bounced GBs contributing to the SCS in at least one $\gamma$ direction, and through which paths. The final result of this first part of the algorithm consists in a list of "Sources" composed of the final image plane, plane identifiers (initial $P_{e}$ and reflecting planes) and frame index lists, and is quite light in terms of 
memory consumption.

In the second part of the algorithm, $S C S(\gamma)$ is calculated for all $\gamma$ directions in a grid of scattering directions.

Only few beams contribute to each $S C S(\gamma)$. Firstly, the opposite of the $\gamma$ direction must belong to the PW spectrum $\widetilde{w}_{\mu_{e}}$ of the original beam source in $P_{e}$. Secondly, $\gamma$ must belong to the scattered beam PW spectrum. The first condition yields an upper boundary $N_{\epsilon}$ for the number of beams per initial spatial origin contributing to $S C S(\gamma)$ with $N_{\epsilon}=\left(2 \Omega^{\epsilon} / \bar{k}\right)^{2}=\frac{-\ln (\epsilon)}{\pi \nu}$ where $\Omega^{\epsilon}$ is the beam spectral extent given in section II-D.

\section{B. Algorithm complexity}

As recalled in [1], the computational complexity of Geometrical Optics to compute monostatic SCS is $O\left(B N^{2}\right)$ with $N=(k R)^{2}$ the usual large parameter and $B$ the maximum number of bounces along ray paths.

The computational complexity of the GBS algorithm detailed above is $O(B N)$ for its first part, for the number of operations is proportional to the number of frame windows needed to span the spatial and spectral domains of fields incident on the $P_{e}$ planes, multiplied by the number of bounces per launched beam. For its second part, its computational complexity is $O(N)$ if $L$ is kept the same for increasing frequencies. With such choice, the number of frame spatial indices is independant from frequency. Knowing that the number of beams per initial spatial origin, contributing to $S C S(\gamma)$, is upper bounded by the $N_{\epsilon}$ constant, independent of frequency, the number of operations for each $\gamma$ direction is $O(1)$. The complexity of this part of the GBS algorithm is thus $O(N)$, proportional to the number of scattering directions required for sufficient sampling of the $3 \mathrm{D}$ angular space.

The global complexity of this algorithm is then $O(B N)$.

\section{Numerical illustration}

This algorithm has been used to compute the monostatic SCS of the environment presented in Fig. 1, with dimensions $S=25 \mathrm{~m}, W=20 \mathrm{~m}, H=24 \mathrm{~m}$, for the $\lambda=1 \mathrm{dm}$ wavelength. The chosen frame parameters were $L=40 \lambda$ and $\nu=0.25$, and the threshold parameter $\epsilon=10^{-3}$.

When performing the GB tracking part of the algorithm, for one of the "vertical" $P_{e}$ planes (e.g. the $x O z$ plane in Fig. 1), the initial number of frame windows spanning all incident PWs directions, and the rectangle of dimensions $H(2 S+W)$ in $P_{e}$, is of the order of $10^{7}$. At the end of the beam tracking part of the algorithm, the number of contributing beams is reduced to about $2 \cdot 10^{4}$. By comparison, a Shooting and Bouncing Rays algorithm with initial sampling of three ray origins per wavelength would lead to about $1.5 \cdot 10^{6}$ rays to shoot, per incident PW.

Fig. 4 shows which indices are eligible for SCS computation, for initial source windows horizontally centered in the "street" aperture (spatial index $m=0$ ) and for all vertical positions of their centers ( $p \in[0: 11]$ ). The eligible beams (yellow or light grey pixels) are not more than $7 \times 7$, and often less, for a given window center, from an initial number of

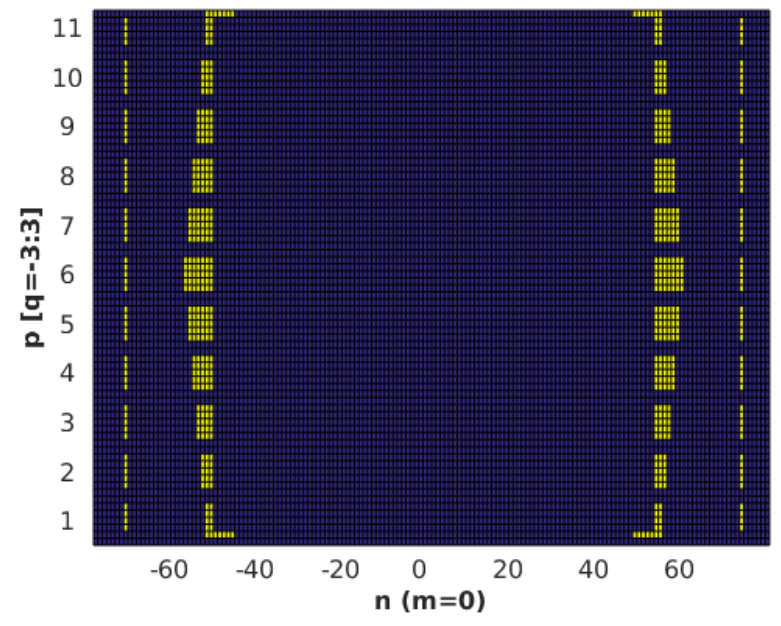

Fig. 4. Eligible beam indices $\mu_{e}=(m, n, p, q)$ for $m=0$.

$159 \times 159$. Each block of adjacent eligible beams corresponds to a common "path" (same successive reflecting surfaces). The dark region at the center of the figure corresponds to beams which are not tilted enough to bounce on the "walls" on each side of the "street". Among all eligible beams, less than $10^{3}$ finally contribute to SCS in each scattering direction, in the second part of the algorithm.

\section{ACCURACY AND VALIDATION METHODOLOGY}

\section{A. Frame parameter influence}

The choice of the frame parameter $L$ is contrained by two contradictory conditions which influence the accuracy of the method. Firstly, $L$ must be large enough to guarantee that $P$, the longest beam path from source $P_{e}$ to last reflexion, is not larger than a few times the beam collimation distance $b=L^{2} / \lambda$. Secondly, $L$ should be small enough, as compared to the linear size of reflecting surfaces or intervals between blocks, so that the error related to inaccurate representation of beam transformation along the block edges is limited to a small fraction of reflected beams. Increasing initial frame redundancy, by decreasing the $\nu$ factor, yields better accuracy of final result [2], but at the expense of computational efficiency.

For the same test scenario as in the previous subsection (III-C), the longest path length is of about $250 \mathrm{~m}$ and the collimation distance $b$ is equal to $160 \mathrm{~m}$. The first condition is thus satisfied. On the other hand, $L / W=0.2$. Better compromises can be established for smaller wavelengths. At $\lambda=1 \mathrm{~cm}$, for $L=80 \lambda, L / W=0.04$ and $b=64 \mathrm{~m}$. Fig. 5 presents the SCS pattern computed along a half circle in the "horizontal" plane ( $x O y$ in Fig. 1). The large parameter $N$ is approximately equal to $4 \cdot 10^{9}$ and the computation time on a single processor machine was less than 30 minutes (less than 30 seconds for the GB tracking part).

Numerical comparisons of fields scattered by reflecting faces with side lengths $a$ and $b$ illuminated by incident PWs, calculated by the simple GBS presented here, and by PO 


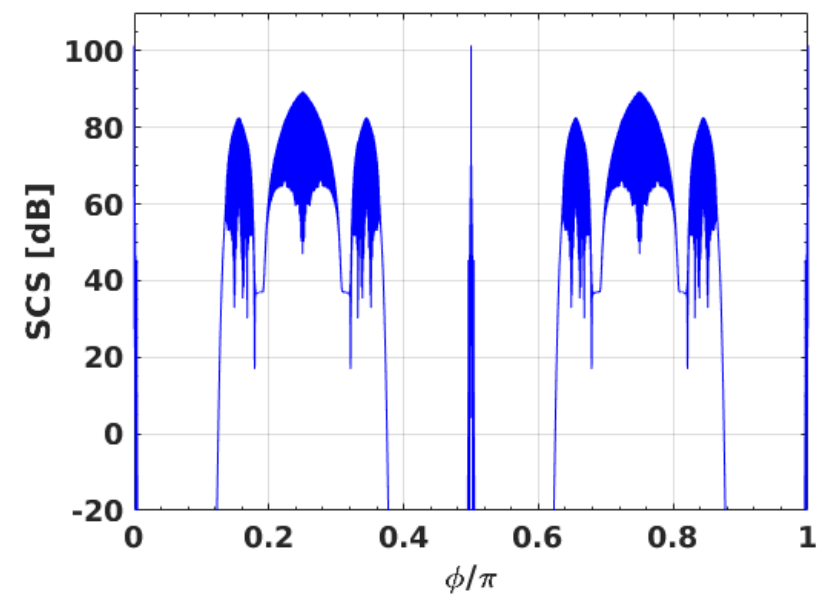

Fig. 5. Monostatic scattering pattern on half circle in $x O y$ plane at $\lambda=1 \mathrm{~cm}$.

or iterative PO [3], will be presented at the conference as functions of $L / a$ and $L / b$ ratios, for one bounce and small numbers of successive bounces.

\section{B. Validation using $2 D$ to $3 D$ conversion}

Towards validation of the SCS computed by the GBS technique, we sought to compare it to a numerically rigorous solution of the problem at hand. Such comparison, however, encounters an inherent difficulty. On one hand, the GBS technique is expected to be accurate only when the scatterer is very large compared to the wavelength. While on the other, the numerically rigorous methods are limited in their ability to model very large 3D problems due to their high computational complexity. To that end, we approximately consider the problem under study (see Fig. 1) as a finite segment of a 2D configuration depicted in Fig. 6. The 2D problem is assumed infinite in the $z$-direction and can be conveniently analyzed using the conventional method of moments (MoM). Since the scatterer comprises four closed surfaces, the Combined Field Integral Equation (CFIE) formulation is chosen to avoid spurious resonances. The 3D monostatic SCS for horizontal angles of incidence $(\theta=\pi / 2)$ can be approximately predicted based on the $2 \mathrm{D}$ one as [4]:

$$
\operatorname{SCS}(\theta=\pi / 2, \phi)=\frac{H^{2}}{2 \pi \lambda} \sigma_{2 D}(\phi)
$$

where $\sigma_{2 D}(\phi)$ denotes the 2D monostatic radar cross section (echo width) at azimuthal angle $\phi$. Conversion formula (5) is based on the assumptions of plane wave incidence and that the equivalent currents induced on the 2D scatterer are simply truncated to height $H$ in the 3D problem. The latter assumption is expected to become inaccurate for complex scattering phenomena involving multiple reflections. Specifically, the 2D MoM and GBS computation results are compared for $\lambda=10 \mathrm{~cm}$. The MoM employs $\lambda / 10$ discretization resulting in 40,000 unknowns. The solution was effected by direct solution of the linear system on a server requiring roughly $50 \mathrm{~GB}$ of

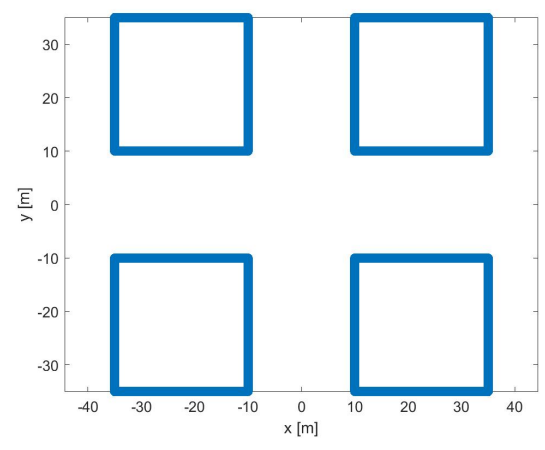

Fig. 6. 2D configuration of the scattering scenario.

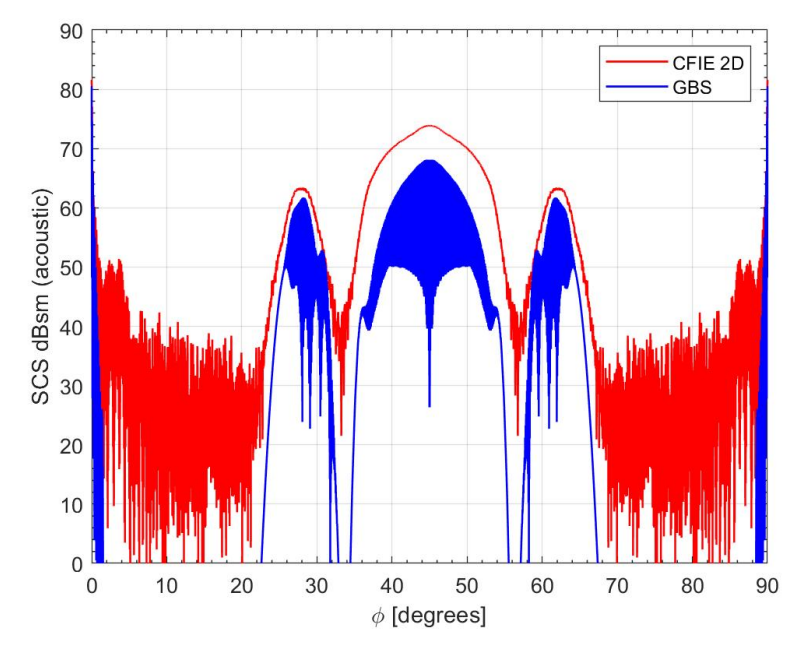

Fig. 7. Comparison between 2D-to-3D MoM and frame based GBS results.

RAM. The resulting SCSs computed using the GBS technique with parameters given in section III-C and MoM followed by the 2D-to-3D conversion are presented in Fig. 7 for the $\phi=0$ to $\pi / 2$ domain. Here, one can observe good agreement for main peaks produced by single scattering events, around $\phi=0$ and $\phi=\pi / 2$. On the other hand, as expected, 2D computations overestimate the multiple scattering phenomena, since in 3D part of the energy is escaping the scatterer in the $z$-direction. One can also notice, that weaker contributions probably due to diffraction effects are not accounted for by this version of the GBS technique and will be a subject of its further extensions.

\section{REFERENCES}

[1] C. Letrou, I. Gershenzon, M. Hariz, and A. Boag, "Fast beam-based analysis of monostatic scattering," in ICEAA 2017 : 19th International Conference on Electromagnetics in Advanced Applications, I. C. Society, Ed., Los Alamitos, 2017, pp. 810-811.

[2] D. Lugara, C. Letrou, A. Shlivinski, E. Heyman, and A. Boag, "Framebased Gaussian beam summation method: Theory and application," Radio Science, vol. 38, no. 2, Apr. 2003.

[3] I. Gershenzon, Y. Brick, and A. Boag, "Shadow radiation iterative physical optics method for high-frequency scattering," IEEE Transactions on Antennas and Propagation, vol. 66, no. 2, pp. 871-883, Feb 2018.

[4] E. Knott, J. Schaeffer, and M. Tulley, Radar Cross Section, 2nd ed. Science Tech Publishing, 2004. 